\title{
SICK BUILDING SYNDROME: NATURAL DAYLIGHT CASE STUDY
}

\author{
Shabnam Akbari Namdar ${ }^{1}$ and Solmaz Tabe Afshar ${ }^{\star 凶}$ \\ ${ }^{1}$ Assistant Professor, Department of Architecture, Islamic Azad University, Tabriz, Iran \\ ${ }^{2}$ PhD student, Department of architecture, Tabriz Branch, Islamic Azad University, Tabriz, Iran
}

\section{Research Article \\ PII: S238315531900005-8}

Received: o8 Aug. 2019 Revised: 25 Nov. 2019 Published: 15 Dec. 2019

Corresponding author's Email: s.tabeafshar@gmail.com

\begin{abstract}
In the taxonomy of creatures, human is a complex being, a feature that makes him vulnerable, such that if he lacked the power to reason and intelligence, he would certainly not be able to deal with the threats of the nature. Creating a shelter, from its primitive form for cave dwellers to today's luxurious buildings, all point to the man's need for privacy that primarily guarantees his security, but a closer look at this physical boundary between man and nature, which is known as residence, reveals that there are a plethora of problems, questions, and requirements besides security, ranging from the extent to which nature suffers as a result of construction to the health problems that this demarcation creates for humans. Evidently, not being attentive to the environment will result in health problems, but since sustainable architecture is not necessarily considered a moral obligation to care for the health of residents, developing building regulations and design policies with an emphasis on human health seems essential. One of such problems, whose consequences are clearly noticeable in current societies and families, is the lack of access to natural daylight as a result of increased unorganized constructions and regardless of environmental conditions, and ultimately the emergence of dysfunctional buildings for their residents in the community, which in turn will eventually bring about irreversible physical and mental problems. In this article, the notion of "Sick Building Syndrome" (or SBS for short), and its underlying causes are reviewed by conducting a series of studies on authoritative and up-to-date sources, articles, and books. Here, the role that natural daylight plays as one of the major elements whose lack or absence will lead to the formation of a sick building in society, along with the factors and elements contributing the increased prevalence of this type of building are studied in the scope of architectural designing. The authors believe that identifying the reasons for the rise of acute health problems in our current society and promoting builders and architects to use appropriate and low-cost solutions, will greatly alleviate these problems.
\end{abstract}

KEYWORDS: Sick Building Syndrome, SBS, Daylight, Architecture \& Health, Architectural Design

\section{INTRODUCTION}

The term "syndrome" is derived from the Greek language, essentially meaning concurrence, while today, it mostly refers to a group of symptoms which consistently occur together [1].

Sick Building Syndrome (SBS) was recognized as a medical condition by the World Health Organization in 1982. The World Health Organization defines SBS as a group of nonspecific symptoms including symptoms such as eye, nose and throat irritation, mental fatigue, headache, nausea, dizziness and skin irritations among other, some of which appear to be related to working or living building [2].

SBS should not convey the notion that the building itself is sick, but that the people in it may have one, some, or all of the symptoms that falls into SBS as per the definition offered by World Health Organization. Sick Building Syndrome is an umbrella term used to describe situations in which occupants of the building experience discomfort and even severe health problems proportional to the duration of residence in the building, even when a specific illness or cause is not identifiable [2].

SBS is often related to the problems in indoor air quality, among which the most effective factors are often in fact a combination of potential factors, including indoor air pollution, lack of sunlight and daylight, poor ventilation and heating, poor acoustics and the presence of pathogenic elements such as asbestos. Biological contaminants are also of concern, for example, lack of sunlight along with high humidity provides grounds for the formation of molds and fungal contaminations [3].

If one wants to achieve optimality in design, harmony with nature should be primarily pursued, where humankind first found its origin a few thousand years ago. In the meantime, sunlight and daylight are heavily involved in keeping us in touch with nature while inside the building. Although this may seem simple and obvious at first, it would be 
very important to know that many of the health problems caused for building occupants may be the result of the underestimation of the significance of daylight by urban designers and architects. Thus, it seems that not carefully considering the basic design accounting for natural light, especially in metropolitan areas, is the primary cause a wide degree of stresses, increased number of some acute diseases, reduced work and educational productivity and even increased duration of hospital stay [4].

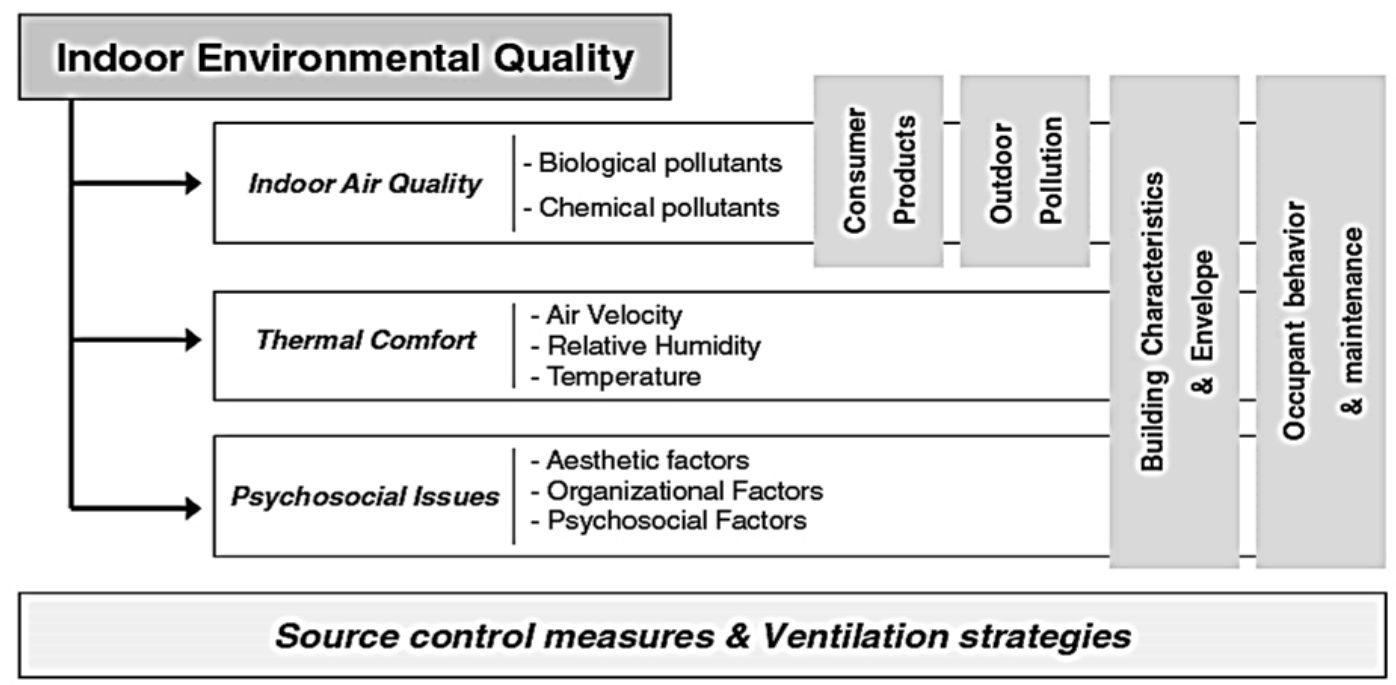

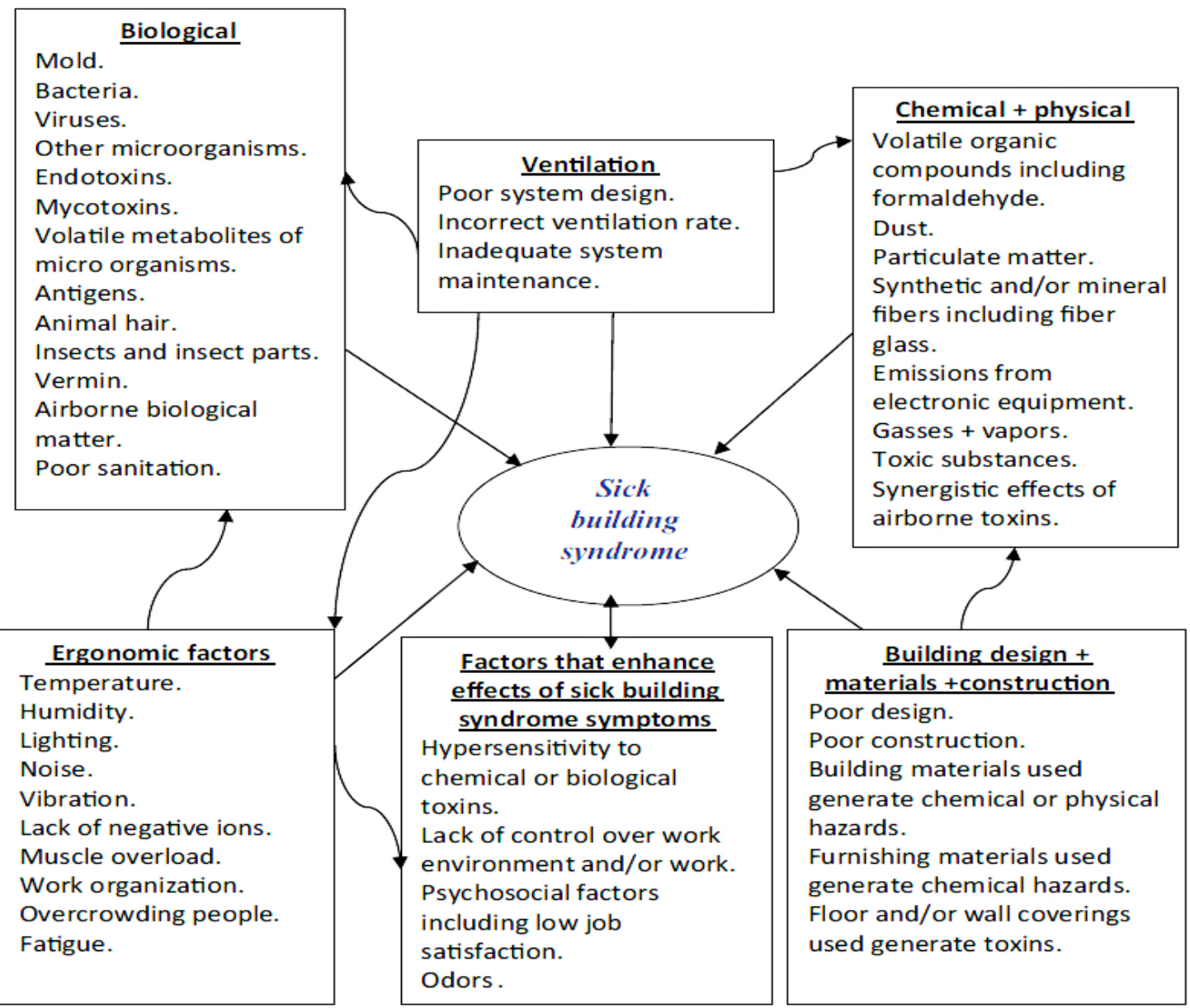

Model of causes of sick building syndrome. 


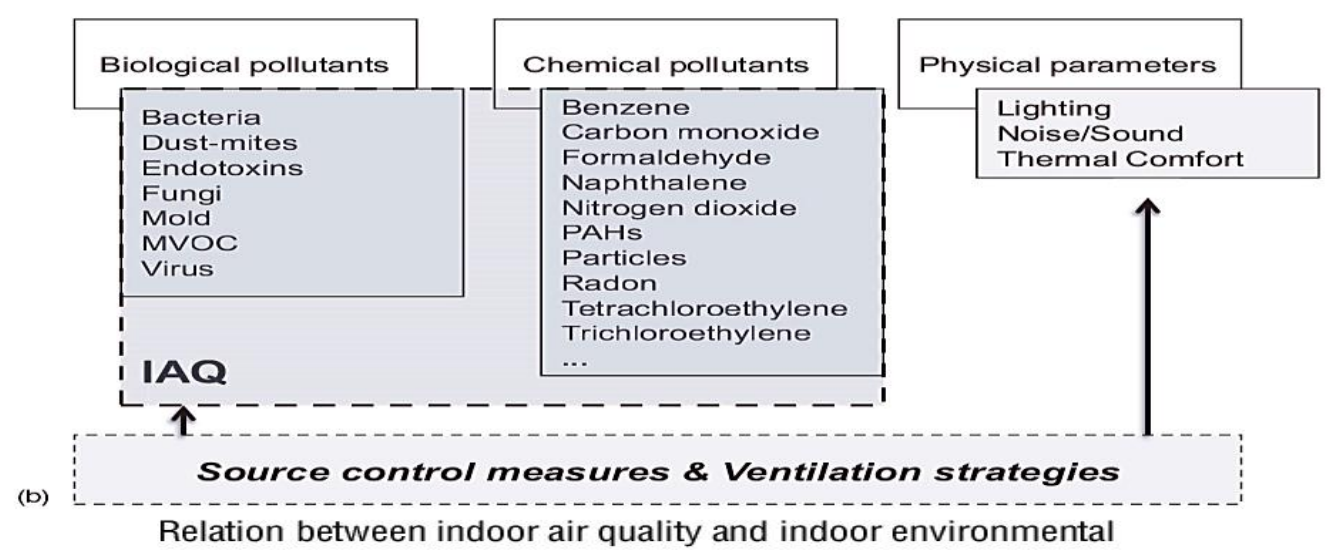

Figure 1. Source control measures and ventilation strategies; Model of causes of sick building syndrome; Relation between indoor air quality and indoor environmental

\section{Reduced employee productivity}

It is generally acknowledged that humans are in better mood during daylight, as most surveys on employees and office buildings show that employees prefer windowed environments to windowless ones. During sunlight, humans feel energetic and lively, but during the winter days we feel bored and even melancholy [5].

\section{Decreased performance and learning in educational environment}

Devotion to environmental quality factors in the designing academic educational spaces has a great impact on the performance of the main users of these spaces, i.e. students. In this sense, one of the most effective environmental quality factors is the introduction of daylight in the designing of educational classes [6].

\section{Delays in patient recovery rate}

In the mid-twentieth century, the famous English nurse Florence Nightingale was one of the major proponents of increasing the amount of natural daylight (sunlight) in homes and wards. She acknowledged that patients admitted to the well-lit wards were in higher morale and much happier than those admitted towards without proper daylight. Known as The Lady with The Lamp, she went so far as to offer architectural designs for low-rise hospital wards that received sunlight not from one direction but from two, thus achieving access to sunlight in the entire ward space [7].

The relationship of humankind with sunlight and natural light throughout history has often manifested in the form of religion and mysticism, such as belief in the sun god in ancient Egypt, which in some cases can be seen quite clearly on stone walls and masonry structures and even caves, although the subject of daylight in its rational form entered the architectural realm from the late nineteenth and early twentieth centuries. Aside from the necessity to preserve energy and protect fossil fuels, the issue of sunlight gained more significance when Dr. Niels Finsen received the 1903 Nobel Prize in Medicine for proving that sunlight could cure tuberculosis. He also developed a method for treating cutaneous tuberculosis using ultraviolet light. Yet the remaining fact is that the light factor with a focus on human health is still often disregarded in design.

\section{Daylight and natural sunlight}

Visible light is a spectrum of electromagnetic waves that is visible to the human eye, varying from 405 terahertz bordering the red light, to 790 terahertz on the purple light. The human body has evolved in the daily cycle of light and darkness and has fully adapted to natural light, which is the result the visible light spectrum being combined, though the sensitivity of the human eye and even skin to any of the colors of the visible light spectrum is different. More importantly, daylight is not a constant flow of light, but a dynamic thing that is a function of time and place and contains information about the outside environment, including the situation of the day and the extent of coldness and heat. Therefore, the spectral quality of sunlight, which cannot be accurately produced artificially, makes it an entirely different type of light compared to what is produced by any electrical source [7].

\section{Daylight and health}

The relationship between health and daylight can be studied from both mental and physical perspectives. Natural light carries information regarding the day. This light gives us information 
about the outside environment when we are indoors because our body is involved in daily shifts throughout the year and this circadian cycle directly affects the digestive system, sleep pattern, hormone secretion and even body temperature. The integrity of the body's biological clock is closely attributed to the amount of light it receives from the environment during the day. As a result, receiving enough daylight is very important for reducing stress, as it affects people's mental states [7].

People became more attentive to the importance of daylight when Dr. Niels Finson received the 1903 Nobel Prize for proving that sunlight could, in fact, cure tuberculosis. In 1984, Dr. Rosenthal and colleagues discovered that the improvement in their patients' depression was more due to sunlight than to the inhibition of the release of the melatonin hormone, as inhibiting the release of this hormone through prescription drug did not reduce depression.

It has been proven that, physically, sunlight is the main source of vitamin D. The medicinal and therapeutic properties of vitamin D were identified at the beginning of the twentieth century. The deficiency of this vitamin at first will lead to a decrease in bone density and hence rickets. Furthermore, there is a direct relation between vitamin $\mathrm{D}$ deficiency and the function of human cardiovascular system. In fact, vitamin D deficiency is an environmental factor that, regardless of the genetic factor, leads to heart failure and high blood pressure in people. Increased rates of high blood pressure in winter compared to summer is potentially owing to reduced levels of vitamin $\mathrm{D}$ in the body as a result of reduced exposure to sunlight. Although there is a plethora of dietary supplements for compensating for vitamin D deficiency, sunlight has been shown to be the main source of vitamin D, which no supplement or diet can replace it [8].

Moreover, a relation between vitamin D deficiency and an increased risk of various cancers has been previously shown. However, it should be noted that depending on the quality, time and place of sunlight, its effect can be either positive or negative. For example, excessive exposure to direct sunlight in a hot and dry climate can increase the risk of skin cancer. Sunlight provides, through photosynthesis and other processes, the chemical elements needed for our survival that are dependent on light. There are fundamental biological, hormonal, and physiological functions within beings that are synchronized through various cycles and are essential for the life of cells, plants, animals, and humans. When there is no sunlight, disruption of the daily life cycle leads to the emergence and spread of diseases and abnormal behaviors in many plants, animals and humans. According to Salamat News, as quoted by ISNA, deficiency and insufficiency of vitamin $\mathrm{D}$ is common in approximately 30 to $50 \%$ of the world's population, although its prevalence varies in different geographical areas and varying nutritional and climatic conditions. Despite medical advances, vitamin $\mathrm{D}$ deficiency is still highly epidemic. 50 to $95 \%$ of the vitamin D in the body is supplied to the skin through sunlight, while the rest is supplied through food and supplements [8].

\section{Urban design policies and designing requirements}

A healthy person is not necessarily someone who is free of any disease, but health is actually being in a balanced state and appropriate physical, mental and social condition. Therefore, providing a living environment that meets the health requirements of residents should be considered with higher priority compared to visual criteria. Based on the aforementioned discussion, the acceptable level of daylight often manifests as one of the important factors of SBS within healthcare in the two scopes of urban design and architecture [9].

In the field of architecture, in most buildings, the issue of light and health is mostly overlooked and visual criteria are often valued higher. Nowadays, there is practically no requirement set for architects in this regard, as most of the criteria are associated with the safety of openings and exits. A severe lack of standardized criteria for improving the health of residents is more visible in this field than in the field of urban design and, of course, is accompanied with more complexity. It is trivial that daylight affects human mood, yet its extent and type depend on the very same human. No any two people see a building and a room in the same way, so despite the fact that there is no alternative to the architect's understanding of the needs and desires of people who want to live, work or play in a specific building, there must be climate-appropriate standards that on the one hand offer information on changes in the 24hour cycle, and on the other hand give residents the ability to control their environment. Well-structured and consistent rules, standards, and guidelines that can also be generalized offer the architect reliable starting platforms in the design process. Criteria for the placement of stairs and emergency exits should be devised in line with the orientation of the building, the location of the bedrooms and living rooms, the depth of the rooms as well as the size and location of windows and shutters and the use of 
special techniques with the purpose of providing the desired natural light inside the building. Having in mind that dazzling and excessive light is also annoying to residents and can even reduce visibility and increase health risks, the key point in designing based on daylight is to control lighting levels as well as control the direction of radiation and even the manner of light distribution by its occupants.

There are strategies in relation to the provision of indoor lighting through side windows, casements or skylights that the architect should consider in accordance with the particular design of the building. The corresponding methods include engineering the size and slope of the side windows and openings, the use of attic side windows with no visibility from outside, reflecting platforms in the side windows for reflecting light from the ceiling to the depth of the room, light guide towers, prismatic system employing light refraction and reflection mechanism, one-way ceiling monitors for incandescent light of winter, light tube system for directing sunlight to the lower floors in multi-story buildings, as well as intelligent systems for adjusting indoor lighting using sunlight. In any case, this issue requires the creativity of the architect, as it must be classified in the construction design standards [9].

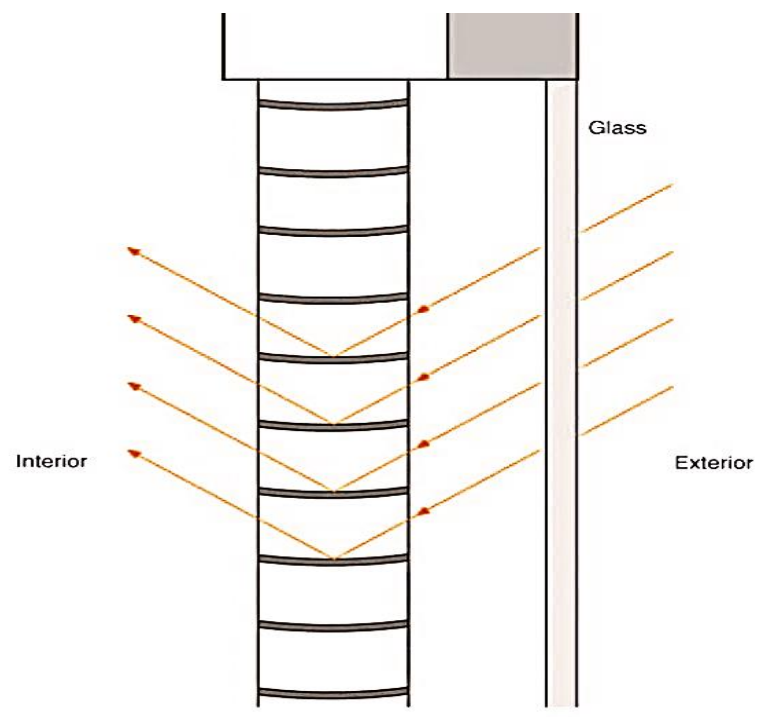

Figure 2. Light-redirecting louver system.

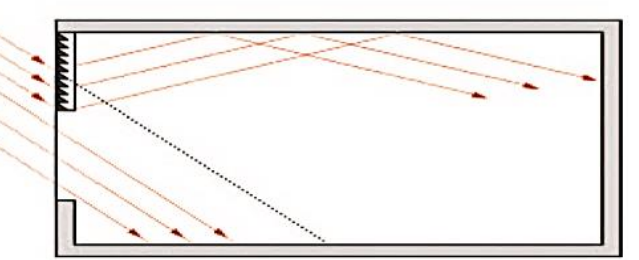

Figure 3. Prismatic panel inserted within a side window redirecting incoming sunlight.

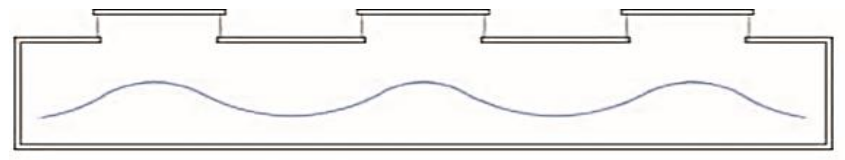

Figure 4. Two sided roof monitor system.

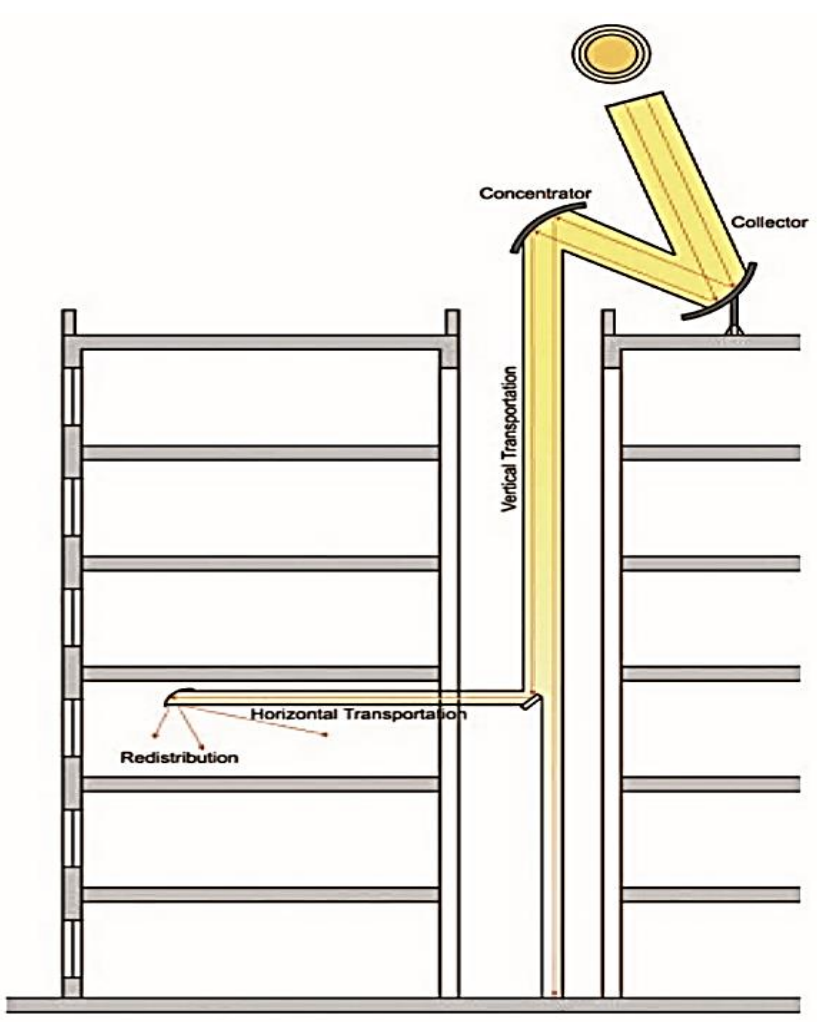

Figure 5. Alight pipe system with its various sunlight collection and light transport systems.

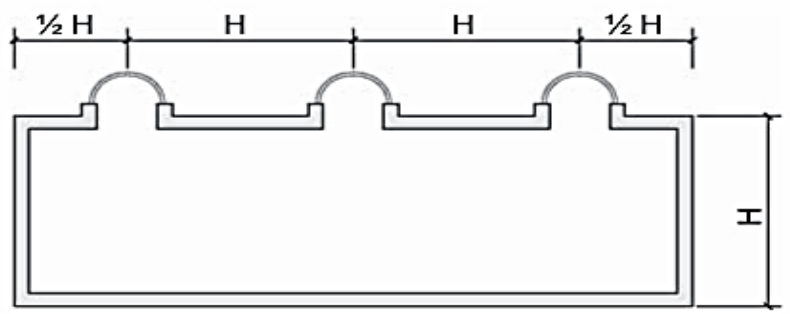

Figure 6. Rule of thumb for spacing skylights to obtain uniform light distribution beneath multiple skylights.

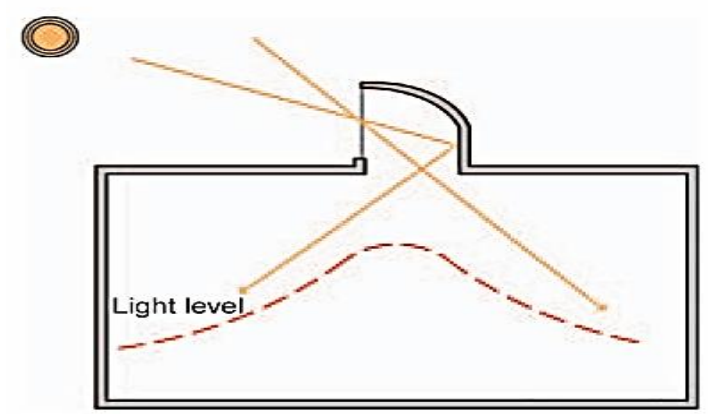

Figure 7. A single-sided roof monitor system designed to allow winter sunlight to enter but not summer sunlight. 


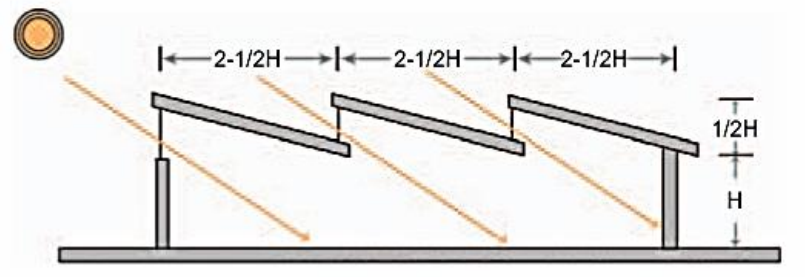

Figure 8. A single sided sawtooth system provides directional distribution of daylight inside the room.

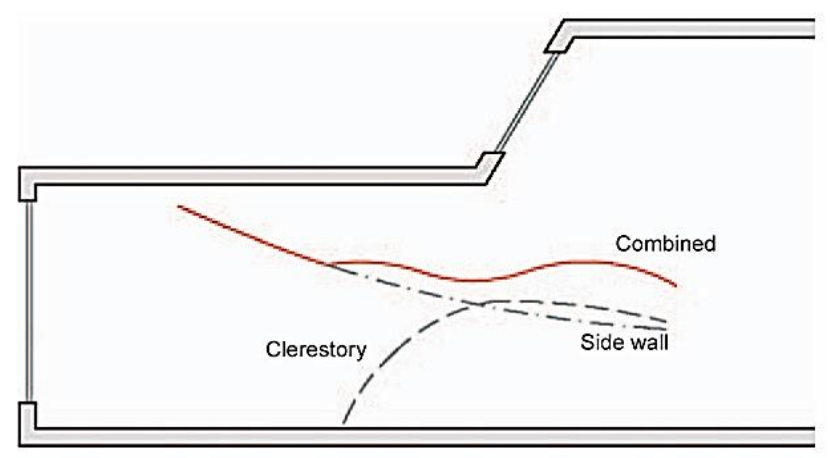

Figure 9. Daylight penetration resulting from the combination of an oblique clerestory and a side window.

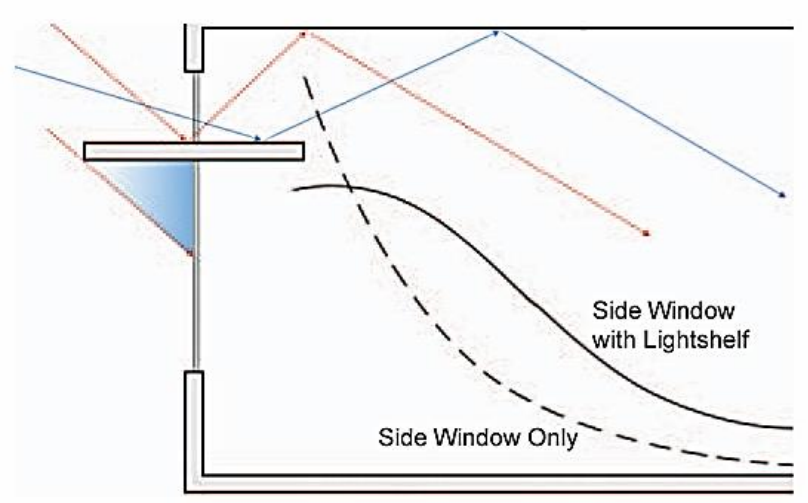

Figure 10. Daylight penetration from a combined lightshelf system.

In spite of all these solutions, designers may not be able to provide daylight in all parts of the building, and hence they can embed daylightcatching rooms, balconies, atriums and terraces in their design, where the occupants of the building can access direct sunlight.

\section{CONCLUSION}

It is evident that with the development of new industrial cities, various mental and physical disorders related to the living environment are emerging, problems that can be identified as a combination of factors under the notion of Sick Building Syndrome (SBS). Daylight is one of the most significant of such factors, the presence or absence of which affects the mental and physiological health of the occupants of the building and their overall health in various ways. Natural daylight through windows and various forms of openings provides the connection for occupants trapped in buildings with the outside space, allowing them to closely sense the nature. Lack of sunlight can even be literally considered a poison as it leads to reduced levels of vitamin $\mathrm{D}$ in human body. Although severe SBS problems are often associated with the duration of time residents stay indoors and will gradually alleviate after leaving the building, not being attentive to this issue may lead to a plethora of severe problems such as heart failure, vascular complications and even the emergence of some serious diseases, as people nonetheless spend a significant share of their lives indoors.

An expert look at the manner of construction in the country reveals that the issue of daylight and health in design in most cases has fallen victim in expense to mass production and economic greed of estate owners. Although a significant share of this problem can be attributed to regulatory problems, more importantly, the development of criteria focusing on the health of residents, accompanied with a solid administrative guarantee, seems to be the key to solving this problem. In this regard, regulation and criteria related to urban design and architecture policies should be included as standards and instructions in the agenda of activists of housing and urban development.

Furthermore, an architect should use natural daylight management strategies inside buildings, considering the parameters of direction, intensity, distribution and controllability in the process with the aim of optimizing design. In this sense, not issuing work permits for non-professionals and having continuous monitoring of design and implementation processes can prove be very promising.

\section{DECLARATIONS}

\section{Authors' contribution}

All the authors contributed equally to the work presented.

\section{Competing interests}

The authors declare that there is no competing interests.

\section{REFERENCES}

[1] Mahmoudi M (2016). Sick Building Syndrome, Springer International Publishing Switzerland,.DOI: 
https://link.springer.com/chapter/10.1007/978-3319-30835-7 29 Google Scholar

[2] Jansz J (2017). Sick Building Syndrome, Elsevier International Encyclopedia of Public Health (Second Edition),6,4-8, DOI: http://dx.doi.org/10.1016/B978-0-12-8036785.00407-0

[3] Bahubail M A (2013). Sick Building Syndromes and Their Effects on Homes within Riyadh City, Department of Architecture and Building Sciences, College of Architecture and Planning, King Saud University.,Vol.25,Arch \&\& Planing (2), pp .69-78, Riyadh(2013/1434H.) Google Scholar

[4] Sabah A, Abdul-Wahab (2011). Sick Building Syndrome in Public Building and Workplaces Google Scholar https://www.springer.com/gp/book/9783642179181

[5] Tregenza P, Wilson M (2011). Daylighting Architecture and Lighting Design. London: Routledge Taylor and Francis Group Google Scholar
https://books.google.com/books?id=viOm6cKMeGkC \&source $=$ gbs book other versions

[6] Ashrafi E, Sadeghi Naeini H (2016). Determination of Effective Factors on Reduction of Sick Building Syndrome in Designing Educational Environment, International Journal of Advanced Biotechnology and Research, 7,144-152 Google Scholar, https://bipublication.com/files/20160419seyede.pdf

[7] Boubekri M (2007). Daylight, Architecture and People's Health. WIT Transactions on Biomedicine and Health, 11 (6) Google Scholar DOI: 10.2495/EHRo70061

[8] Naibi, B (2007). The effect of indoor lighting on quality of life and human moral behaviors, Quarterly Journal of Ethics in Science and Technology, 2007, Nos. 3 and 4

[9] Boubekri M (2008). Daylight, Architecture and Health Building Design Strategies, Elsevier. Google Scholar

https://www.sciencedirect.com/book/978075066724 1/daylighting-architecture-and-health 